\title{
COHOMOLOGY OF THE LIE ALGEBRAS OF DIFFERENTIAL OPERATORS: LIFTING FORMULAS
}

\author{
BORIS SHOIKHET
}

\section{INTRODUCTION}

The lifting formulas are formulas for the cocycles of the Lie algebra $\mathfrak{A}$, constructed from an associative algebra $\mathfrak{A}$ with the bracket $[a, b]=a \cdot b-b \cdot a$. These cocycles are constructed with the following data:

1) a trace $\operatorname{Tr}: \mathfrak{A} \rightarrow \mathbb{C}$ on the associative algebra $\mathfrak{A}$

2 ) a set $\mathcal{D}=\left\{D_{1}, D_{2}, \ldots\right\}$ of the (exterior) derivations of the associative algebra $\mathfrak{A}$ satisfying the following conditions:

(i) $\operatorname{Tr}\left(D_{i} A\right)=0$ for any $A \in \mathfrak{A}$ and any $D_{i} \in \mathcal{D}$

(ii) $\left[D_{i}, D_{j}\right]=\operatorname{ad}\left(Q_{i j}\right)$ - inner derivation $\left(Q_{i j} \in \mathfrak{A}\right)$ for any $D_{i}, D_{j} \in \mathcal{D}$

(iii) $\operatorname{Alt}_{i, j, k} D_{k}\left(Q_{i j}\right)=0$ for all $i, j, k$.

The main example of such a situation is the Lie algebra $\Psi \operatorname{Dif}_{n}\left(S^{1}\right)$ of the formal pseudodifferential operators on $\left(S^{1}\right)^{n}$ (see [A] ). The trace Tr in this example is the "noncommutative residue", $\operatorname{Tr}(D)=$ the coefficient of the term $x_{1}^{-1} \cdot x_{2}^{-1} \cdot \ldots \cdot x_{n}^{-1} \cdot \partial_{1}^{-1} \ldots \cdot \partial_{n}^{-1}$ of $D \in \Psi \operatorname{Dif}_{n}\left(S^{1}\right)$ (in any coordinate system). It is easy to verify that $\operatorname{Tr}\left[D_{1}, D_{2}\right]=0$ for any $D_{1}, D_{2} \in \Psi \operatorname{Dif}_{n}\left(S^{1}\right)$. Furthermore, $\ln x_{i}(i=1, \ldots, n)$ are (exterior) derivations of $\Psi \operatorname{Dif}_{n}\left(S^{1}\right)$ with respect to the adjoint action; the symmetry between the operators $x$ and $\frac{d}{d x}$ allows us to define exterior derivations $\ln \partial_{i}(i=1, \ldots, n)$. (Actually, one can define $\ln D \quad\left(D \in \Psi \operatorname{Dif}_{n}\left(S^{1}\right)\right)$ in much greater generality - see Sect. 2.4 and 2.8).

We prove in $\S 1$ that the noncommutative residue $\operatorname{Tr}$ on the associative algebra $\Psi \operatorname{Dif}_{n}\left(S^{1}\right)$ and the set of $2 n$ derivations $\left\{\ln x_{i}, \ln \partial_{i} ; i=1, \ldots, n\right\}$ satisfy conditions (i)-(iii) above, and this is our main (and in some sense the unique) example.

In the case of the one derivation $D$ such a construction appeared in [KK], where two 2-cocycles on the Lie algebra $\Psi \operatorname{Dif}_{1}\left(S^{1}\right)=\Psi \operatorname{Dif}\left(S^{1}\right)$ were constructed:

$$
\begin{aligned}
& \Psi^{(1)}\left(A_{1}, A_{2}\right)=\operatorname{Tr}\left(\left[\ln x, A_{1}\right] \cdot A_{2}\right) \\
& \Psi^{(2)}\left(A_{1}, A_{2}\right)=\operatorname{Tr}\left(\left[\ln \partial, A_{1}\right] \cdot A_{2}\right) .
\end{aligned}
$$

Both these cocycles are cohomologous to zero when restricted to the Lie algebra Dif 1 of the (polynomial) differential operators on $\mathbb{C}^{1}$; on the other hand, our aim is to construct cocycles on this Lie algebra. We are able to accomplish this by the simultaneous application of both $\ln x_{i}$ and $\ln \partial_{i}$.

Now the problem is solved only for $n=1(\S 1)$ and $n=2(\S 3)$. But the Second Version of the Main Conjecture ( $\S 4$ ) gives us an explicit formula for arbitrary $n$ (when conditions (i)-(iii) above hold). We obtain this formulas using a some simple trick, and all that

Date: 1997. 
remains to prove is the fact that this formula gives in fact cocycles. We will see in $\S 3$ $(n=2)$ that this is very difficult problem.

In this way we obtain elements in $H^{2 n+1}\left(\operatorname{Dif}_{n} ; \mathbb{C}\right)$. We assume that $H^{2 n+1}\left(\operatorname{Dif}_{n} ; \mathbb{C}\right)=$ $\mathbb{C}$ (but we don't use this fact elsewhere). There is a deformation of the Lie algebra Poiss $_{2 n}$ of the functions on $\mathbb{R}^{2 n}$ with the Poisson bracket to the Lie algebra Dif ${ }_{n}$, and the well-known result is the vanishing theorem: $H^{i}\left(\operatorname{Poiss}_{2 n} ; \mathbb{C}\right)=0$ when $0<i \leq 2 n$. Hence, we study the first nontrivial case.

It is easy to construct a $(2 n+1)$-cocycle on $\operatorname{Poiss}_{2 n}$ (or on the Lie algebra $\operatorname{Poiss}_{2 n}\left(S^{1}\right)$ of the functions on the torus $\left(T^{2}\right)^{n}$ with the Poisson bracket), and if the lifting problem can be solved, the corresponding $(2 n+1)$-cocycle on $\Psi \operatorname{Dif}_{n}\left(S^{1}\right)$ is a deformation of this $(2 n+1)$-cocycle on $\operatorname{Poiss}_{2 n}\left(S^{1}\right)$. The lifting problem is equivalent to the deformation problem $(\S 2)$.

B. Feigin explained me that such formulas should exist; numerous talks with him and with M. Kontsevich made me interested in this subject. I express my deep gratitude to them. I am also grateful to Jeremy Bem for helping with my English and to Seva Kordonsky for carefully typing this manuscript.

\section{Cohomology of the Lie algebra Dif 1}

1.1. Let $\operatorname{Tr}=\operatorname{pr}_{x^{-1} \partial^{-1}}$ be a trace on the associative algebra $\Psi \operatorname{Dif}\left(S^{1}\right), D_{1}=\operatorname{ad}(\ln \partial)$ and $D_{2}=\operatorname{ad}(\ln x)$ be two its exterior derivations.

Lemma. (i) $\left[D_{1}, D_{2}\right]=\operatorname{ad} Q\left(Q \in \Psi \operatorname{Dif}\left(S^{1}\right)\right)$ is an inner derivation of the associative algebra $\Psi \operatorname{Dif}\left(S^{1}\right)$.

(ii) $Q=x^{-1} \partial^{-1}+\frac{1}{2} x^{-2} \partial^{-2}+\frac{2}{3} x^{-3} \partial^{-3}+\ldots+\frac{(n-1) !}{n} x^{-n} \partial^{-n}+\ldots$

Proof. It is sufficient to show that $\left[D_{1}, D_{2}\right](x)=[Q, x]$ and $\left[D_{1}, D_{2}\right](\partial)=[Q, \partial]$. This is a straightforward calculation.

\section{Theorem.}

$$
\Psi_{3}\left(A_{1}, A_{2}, A_{3}\right)=\underset{A, D}{\operatorname{Alt}} \operatorname{Tr}\left(D_{1} A_{1} \cdot D_{2} A_{2} \cdot A_{3}\right)+\underset{A}{\operatorname{Alt}} \operatorname{Tr}\left(Q \cdot A_{1} \cdot A_{2} \cdot A_{3}\right)
$$

is a 3-cocycle on the Lie algebra $\Psi \operatorname{Dif}\left(S^{1}\right)$. This cocycle is not cohomologous to zero, and it remains noncohomologous to zero after restriction to the Lie algebra Dif ${ }_{1}$.

We will prove this theorem in Sect. 1.2-1.4.

1.2 .

Lemma. $\operatorname{Tr}\left(D_{i} A\right)=0$ for any $A \in \Psi \operatorname{Dif}\left(S^{1}\right)$ and $i=1,2$.

Proof. It is clear that $\left[\Psi \operatorname{Dif}\left(S^{1}\right), \Psi \operatorname{Dif}\left(S^{1}\right)\right] \quad \in \quad \operatorname{Ker} \operatorname{Tr} D_{i} A$, but $\operatorname{codim}\left(\left[\Psi \operatorname{Dif}\left(S^{1}\right), \Psi \operatorname{Dif}\left(S^{1}\right)\right]\right)=1$. Hence it is sufficient to verify the Lemma for $A=x^{-1} \partial^{-1}$.

\section{Corollary.}

$$
\underset{A, D}{\operatorname{Alt}} \operatorname{Tr}\left(D_{1}\left(D_{2} A_{1} \cdot A_{2} \cdot A_{3} \cdot A_{4}\right)\right)=0
$$

for any $A_{1}, A_{2}, A_{3}, A_{4} \in \Psi \operatorname{Dif}\left(S^{1}\right)$.

The alternation here is just decoration. 
1.3. Let us calculate the 1.h.s. of (1), using the Leibniz rule; we have:

$$
\begin{aligned}
\text { l.h.s }(1)=\operatorname{Alt}_{A, D} \operatorname{Tr}\left(D_{2} A_{1} \cdot D_{1} A_{2} \cdot A_{3} \cdot A_{4}+D_{2} A_{1} \cdot A_{2} \cdot A_{3} \cdot D_{1} A_{4}\right)+ & \\
& +\operatorname{Alt}_{A} \operatorname{Tr}\left(\left[Q, A_{1}\right] \cdot A_{2} \cdot A_{3} \cdot A_{4}\right) .
\end{aligned}
$$

Note that $\underset{A, D}{\operatorname{Alt}} \operatorname{Tr}\left(D_{2} A_{1} \cdot A_{2} \cdot D_{1} A_{3} \cdot A_{4}\right) \equiv 0$ in view of the main property of $\operatorname{Tr}$ and the symmetry conditions,

$$
\text { r.h.s. }(2)=-2 \underset{A, D}{\operatorname{Alt}} \operatorname{Tr}\left(D_{1} A_{1} \cdot D_{2} A_{2} \cdot A_{3} \cdot A_{4}\right)+\underset{A}{\operatorname{Alt}} \operatorname{Tr}\left(\left[Q, A_{1}\right] \cdot A_{2} \cdot A_{3} \cdot A_{4}\right)
$$

Let us denote the first summand in (3) by $\alpha$ and the second one by $\beta$

Lemma. (i) $\alpha=d\left(-2 \underset{A, D}{\operatorname{Alt}} \operatorname{Tr}\left(D_{1} A_{1} \cdot D_{2} A_{2} \cdot A_{3}\right)\right)\left(A_{4}, A_{1}, A_{2}, A_{3}\right)$;

(ii) $\beta=d\left(-2 \operatorname{Alt}_{A} \operatorname{Tr}\left(Q \cdot A_{1} \cdot A_{2} \cdot A_{3}\right)\right)\left(A_{4}, A_{1}, A_{2}, A_{3}\right)$.

Proof. We will use the following expression for the differential in the cochain complex of the Lie algebra:

$$
\begin{aligned}
& \left(d \Psi_{n}\right)\left(A_{1}, \ldots, A_{n+1}\right)=\frac{1}{2} \cdot \sum_{i=1}^{n+1}(-1)^{i-1} \cdot \\
& \cdot\left(\Psi_{n}\left(\left[A_{i}, A_{1}\right], A_{2}, \ldots, \hat{A}_{i}, \ldots, A_{n+1}\right)+\Psi_{n}\left(A_{1},\left[A_{i}, A_{2}\right], \ldots, \hat{A}_{i}, \ldots, A_{n+1}\right)+\ldots\right)
\end{aligned}
$$

Let us prove (i):

$$
\begin{aligned}
& d\left(\underset{A, D}{\operatorname{Alt}} \operatorname{Tr}\left(D_{1} A_{1} \cdot D_{2} A_{2} \cdot A_{3}\right)\right)\left(A_{4}, A_{1}, A_{2}, A_{3}\right)= \\
& =\frac{1}{2} \underset{\substack{A_{1}, \ldots, A_{4} \\
D_{1}, D_{2}}}{\operatorname{Alt}} \operatorname{Tr}\left(D_{1}\left[A_{4}, A_{1}\right] \cdot D_{2} A_{2} \cdot A_{3}+\right. \\
& \left.\quad+D_{1} A_{1} \cdot D_{2}\left[A_{4}, A_{2}\right] \cdot A_{3}+D_{1} A_{1} \cdot D_{2} A_{2} \cdot\left[A_{4}, A_{3}\right]\right) .
\end{aligned}
$$

Then we subtract from r.h.s. of (5)

$$
\begin{aligned}
\gamma=\frac{1}{2} \operatorname{Alt}_{A, D} \operatorname{Tr}\left(\left[A_{4}, D_{1} A_{1}\right] \cdot D_{2} A_{2}\right. & \cdot A_{3}+ \\
& \left.+D_{1} A_{1} \cdot\left[A_{4}, D_{2} A_{2}\right] \cdot A_{3}+D_{1} A_{1} \cdot D_{2} A_{2} \cdot\left[A_{4}, A_{3}\right]\right),
\end{aligned}
$$

(it is clear that $\gamma=0$ ).

We have:

$$
\begin{gathered}
\text { r.h.s. }(5)=\frac{1}{2} \underset{\substack{A_{1}, \ldots, A_{4} \\
D_{1}, D_{2}}}{\operatorname{Alt}} \operatorname{Tr}\left(\left[D_{1} A_{4}, A_{1}\right] \cdot D_{2} A_{2} \cdot A_{3}+D_{1} A_{1} \cdot\left[D_{2} A_{4}, A_{2}\right] \cdot A_{3}\right), \\
\underset{A, D}{\operatorname{Alt}} \operatorname{Tr}\left(D_{1} A_{4} \cdot A_{1} \cdot D_{2} A_{2} \cdot A_{3}-D_{1} A_{1} \cdot A_{2} \cdot D_{2} A_{4} \cdot A_{3}\right)=0
\end{gathered}
$$

in view of the symmetry conditions, and

$$
\text { r.h.s }(5)=\frac{1}{2} \cdot 2 \cdot \underset{A, D}{\operatorname{Alt}} \operatorname{Tr}\left(D_{1} A_{1} \cdot D_{2} A_{2} \cdot A_{3} \cdot A_{4}\right) .
$$

The proof of (ii) is similar. 
1.4. It follows from Corollary 1.2 and Lemma 1.3 that

$$
d\left(\operatorname{Alt}_{A, D} \operatorname{Tr}\left(D_{1} A_{1} \cdot D_{2} A_{2} \cdot A_{3}\right)+\underset{A}{\operatorname{Alt}} \operatorname{Tr}\left(Q \cdot A_{1} \cdot A_{2} \cdot A_{3}\right)\right)=0,
$$

and therefore

$$
\Psi_{3}\left(A_{1}, A_{2}, A_{3}\right)=\underset{A, D}{\operatorname{Alt}} \operatorname{Tr}\left(D_{1} A_{1} \cdot D_{2} A_{2} \cdot A_{3}\right)+\underset{A}{\operatorname{Alt}} \operatorname{Tr}\left(Q \cdot A_{1} \cdot A_{2} \cdot A_{3}\right)
$$

is a 3 -cocycle on the Lie algebra $\Psi \operatorname{Dif}\left(S^{1}\right)$. To complete the proof of Theorem 1.1, it is sufficient to verify that the restriction of $\Psi_{3}$ on Dif ${ }_{1}$ is nonhomologous to zero cocycle. The check is straightforward: $1 \wedge x \wedge \partial$ is a cycle on $\operatorname{Dif}_{1}$ and $\Psi_{3}(1 \wedge x \wedge \partial) \neq 0$.

1.5. In $[\mathbb{F}]$ B. Feigin formulated a conjecture about $H^{*}\left(\operatorname{Dif}_{1} ; \mathbb{C}\right)$. According to this conjecture,

$$
H^{*}\left(\operatorname{Dif}_{1}, \mathbb{C}\right)=\wedge^{*}\left(\Psi_{3}, \Psi_{5}, \Psi_{7}, \ldots\right) \otimes S^{*}\left(c_{4}, c_{6}, c_{8}, \ldots\right)
$$

(the lower index denotes the grading). This conjecture has not been proved yet. However, our methods allow to construct some cocycles $\widetilde{\Psi}_{5}, \widetilde{\Psi}_{7}, \widetilde{\Psi}_{9}, \ldots$ which are "candidates" for $\Psi_{5}, \Psi_{7}, \Psi_{9}, \ldots$; unfortunately, even the proof of their nontriviality runs into trouble because there are not explicit formulas for the cycles. Also, it seems that one can't obtain a proof by passing to the Hamiltonian limit. Note that no representatives for $c_{4}, c_{6}, c_{8}$, ... have been found.

Theorem. For $i=2,3,4, \ldots$

$$
\begin{aligned}
& \widetilde{\Psi}_{2 i+1}\left(A_{1}, \ldots, A_{2 i+1}\right)=\underset{A}{\operatorname{Alt}} \operatorname{Tr}\left(Q \cdot A_{1} \cdot \ldots \cdot A_{2 i+1}\right)+ \\
& +\operatorname{Alt}_{A, D} \operatorname{Tr}\left(D_{1} A_{1} \cdot D_{2} A_{2} \cdot A_{3} \cdot \ldots A_{2 i+1}+D_{1} A_{1} \cdot A_{2} \cdot A_{3} \cdot D_{2} A_{4} \cdot A_{5} \cdot \ldots \cdot A_{2 i+1}+\right. \\
& \quad+D_{1} A_{1} \cdot A_{2} \cdot A_{3} \cdot A_{4} \cdot A_{5} \cdot D_{2} A_{6} \cdot A_{7} \cdot \ldots \cdot A_{2 i+1}+\ldots+ \\
& \left.\quad+a_{s} \cdot D_{1} A_{1} \cdot A_{2} \cdot \ldots \cdot A_{s} \cdot D_{2} A_{s+1} \cdot A_{s+2} \cdot \ldots \cdot A_{2 i+1}\right)
\end{aligned}
$$

where $\left\{\begin{array}{l}s=i+1, a_{s}=\frac{1}{2}, \text { if } i \text { is even } \\ s=i, \quad a_{s}=1, \text { if } i \text { is odd }\end{array}\right.$

is a $(2 i+1)$-cocycle on the Lie algebra $\Psi \operatorname{Dif}\left(S^{1}\right)\left(\right.$ or $\left.\operatorname{Dif}_{1}\right)$.

Proof. It is a direct calculation, similar to the Proof of Theorem 1.1.

Conjecture. $\Psi_{3}, \widetilde{\Psi}_{5}, \widetilde{\Psi}_{7}, \widetilde{\Psi}_{9} \ldots$ generate the exterior algebra in $H^{*}\left(\operatorname{Dif}_{1}, \mathbb{C}\right)$.

Note, that from this Conjecture follows the statement about spectral sequence from [F]. Therefore, if this Conjecture is true, all we need to prove Feigin's conjecture about $H^{*}\left(\operatorname{Dif}_{1}, \mathbb{C}\right)$ is the "main theorem of the invariant theory" for the Lie algebra $\mathfrak{g l}(\lambda)$ see $[\mathrm{F}]$.

\section{The Lifting PRoblem AND the Deformation PROBlem}

2.1. Let $\mathfrak{A}$ be an associative algebra, let us denote by the same symbol the corresponding Lie algebra with the bracket $[a, b]=a \cdot b-b \cdot a$. Suppose that there is an invariant form $\langle$,$\rangle on the Lie algebra \mathfrak{A}$ (not necessary nondegenerate).

For any Lie algebra $\mathfrak{g}$ there is a canonical map $\theta_{i+1}: H^{i+1}(\mathfrak{g} ; \mathbb{C}) \rightarrow H^{i}\left(\mathfrak{g} ; \mathfrak{g}^{*}\right)$. Inner derivations $\operatorname{Inn}(\mathfrak{A})$ of the associative algebra $\mathfrak{A}$ form an ideal in the Lie algebra $\operatorname{Der}_{\text {Ass }}(\mathfrak{A})$ of all derivations of the associative algebra $\mathfrak{A}, \operatorname{Inn}(\mathfrak{A})$ is also an ideal in the Lie algebra 
$\operatorname{Der}_{\text {Lie }}(\mathfrak{A})$ of the derivations of the Lie algebra $\mathfrak{A}$. There is a map $\operatorname{Der}_{\text {Ass }}(\mathfrak{A}) \rightarrow \operatorname{Der}_{\text {Lie }}(\mathfrak{A})$ and a map $\operatorname{Der}_{\text {Ass }}(\mathfrak{A}) / \operatorname{Inn}(\mathfrak{A}) \rightarrow \operatorname{Der}_{\text {Lie }}(\mathfrak{A}) / \operatorname{Inn}(\mathfrak{A})$. The last space is equal to $H^{1}(\mathfrak{A}, \mathfrak{A})$. Let $D_{1}, \ldots, D_{i} \in \operatorname{Der}_{\text {Ass }}(\mathfrak{A})$; we will use the same symbols for their images in $H^{1}(\mathfrak{A} . \mathfrak{A})$. There is a map $H^{m}(\mathfrak{A}, \mathfrak{A}) \otimes H^{n}(\mathfrak{A} ; \mathfrak{A}) \rightarrow H^{m+n}(\mathfrak{A} ; \mathfrak{A})$ because $\mathfrak{A}$ is an associative algebra.

Let $\alpha_{D_{1}, \ldots, D_{i}}=\operatorname{Alt}_{D}\left(D_{1} \cdot D_{2} \cdot \ldots \cdot D_{i}\right) \in H^{i}(\mathfrak{A} ; \mathfrak{A})$ or, in the explicit form, $\alpha_{D_{1}, \ldots, D_{i}}\left(A_{1}, \ldots, A_{i}\right) \stackrel{\operatorname{Alt}_{A, D}}{=}\left(D_{1}\left(A_{1}\right) \cdot D_{2}\left(A_{2}\right) \cdot \ldots \cdot D_{i}\left(A_{i}\right)\right)$. The form $\langle$,$\rangle determines$ the invariant map $\mathfrak{A} \rightarrow \mathfrak{A}^{*}$, and we have the element $\alpha_{D_{1}, \ldots, D_{i}}^{*} \in H^{i}\left(\mathfrak{A} ; \mathfrak{A}^{*}\right)$.

Question. When does there exist an element $\Psi_{i+1} \in H^{i+1}(\mathfrak{A}, \mathbb{C})$ such that $\theta_{i+1}\left(\Psi_{i+1}\right)=$ $\alpha_{D_{1}, \ldots, D_{i}}^{*}$. If such an element exists, it is called lifting of $\alpha_{D_{1}, \ldots, D_{n}}^{*} \cdot\left(\right.$ Note, that $\Psi_{i+1}$, if it exists, is not uniquely determined.)

Furthermore, if $D_{i}^{(j)} \in H^{1}(\mathfrak{A} ; \mathfrak{A}), a_{j} \in \mathbb{C}$, we have an element $\sum a_{k} \alpha_{D_{1}^{(k)}, \ldots, D_{i}^{(k)}}$, and there is the same lifting problem for this element.

2.2. We will be concerned only with the case when there is a trace $\operatorname{Tr}: \mathfrak{A} \rightarrow \mathbb{C}$ on the associative algebra $\mathfrak{A}$ (i.e., $\operatorname{Tr}\left[A_{1}, A_{2}\right]=0$ for any $A_{1}, A_{2} \in \mathfrak{A}$ ) and $\left\langle A_{1}, A_{2}\right\rangle=$ $\operatorname{Tr}\left(A_{1} \cdot A_{2}\right)$. Suppose also, that the condition (i) from the Introduction holds: $\operatorname{Tr}(D A)=0$ for all $A \in \mathfrak{A}$ and $D \in \mathcal{D}=\left\{D_{1}, D_{2}, \ldots, D_{i}\right\}$. Such derivations form an Lie subalgebra $\operatorname{Der}_{\text {Ass }}^{\operatorname{Tr}}(\mathfrak{A}) \subset \operatorname{Der}_{\text {Ass }}(\mathfrak{A})$; we will denote by $H_{\operatorname{Tr}}^{1}(\mathfrak{A} ; \mathfrak{A})$ the image of this subalgebra in $H^{1}(\mathfrak{A} ; \mathfrak{A})$.

Lemma. Suppose that $\sum_{a} a_{k} \cdot D_{1}^{(k)} \wedge \cdots \wedge D_{i}^{(k)}$ is a cycle in Lie algebra $\operatorname{Der}_{\text {Ass }}^{\operatorname{Tr}}(\mathfrak{A})$ (but not only in $\left.H_{\operatorname{Tr}}^{1}(\mathfrak{A} ; \mathfrak{A})\right)$. Then $\Psi_{i+1}\left(A_{1}, \ldots, A_{i+1}\right)=\operatorname{Alt}_{A, D} \operatorname{Tr}\left(\sum_{k} a_{k} D_{1}^{(k)} A_{1} \cdot \ldots \cdot D_{i}^{(k)} A_{i} \cdot A_{i+1}\right)$ is a cocycle, and the corresponding element in $H^{i+1}(\mathfrak{A} ; \mathbb{C})$ is a lifting of $\sum_{k} a_{k} \alpha_{D_{1}^{(k)}, \ldots, D_{i}^{(k)}}^{\left({ }^{(k)}\right.}$.

Proof. It is sufficient to prove that $\Psi_{i+1}$ is a cocycle. As in the proof of Theorem 1.1 we have to write an expression on $A_{1}, \ldots, A_{i+2}$ which is a coboundary and which a priori is equal to zero.

For simplicity we suppose, that $D_{1} \wedge \cdots \wedge D_{i}$ is a cycle in $\operatorname{Der}_{\mathrm{Ass}}^{\operatorname{Tr}}(\mathfrak{A})$.

Consider the following expression:

$$
\underset{A, D}{\operatorname{Alt}} \operatorname{Tr}\left(D_{i}\left(\operatorname{Cycle}\left(D_{1} A_{1} \cdot \ldots \cdot D_{i_{1}} A_{i_{1}} \cdot A_{i} \cdot A_{i+1}\right) \cdot A_{i+2}\right) .\right.
$$

(Here Cycle is the sum on all the cyclic permutations; it contains $i+1$ summands.)

The part of (6) which contains $\left[D_{a}, D_{b}\right]$ is equal to zero because $D_{1} \wedge \cdots \wedge D_{i}$ is a cycle.

The remaining summands are of 3 types:

$$
\begin{array}{r}
\operatorname{Alt} \operatorname{Tr}(D \cdot D \cdot \ldots \cdot D \cdot A \cdot D \cdot D \cdot A), \\
\operatorname{Alt} \operatorname{Tr}(D \cdot D \cdot \ldots \cdot D \cdot D \cdot A \cdot D \cdot A), \\
\text { and } \operatorname{Alt} \operatorname{Tr}(D \cdot D \cdot \ldots \cdot D \cdot D \cdot D \cdot A \cdot A) .
\end{array}
$$

(Here $A$ denotes $A_{a}$ and $D$ denotes $D_{b} A_{a}$.) The summands of the first two types are not coboundaries, but the summands of the third type are. Therefore we have to eliminate the summands of the first two types. 
We have:

$$
\begin{aligned}
& (6)=\underset{A, D}{\operatorname{Alt}} \operatorname{Tr}\left(\operatorname{Cycle}\left(D_{1} A_{1} \cdots D_{i-1} A_{i-1} \cdot D_{i} A_{i} \cdot A_{i+1}\right) \cdot A_{i+2}\right)+ \\
& +\operatorname{Alt}_{A, D} \operatorname{Tr}\left(\operatorname{Cycle}\left(D_{1} A_{1} \cdots D_{i-1} A_{i-1} \cdot A_{i} \cdot D_{i} A_{i+1}\right) \cdot A_{i+2}\right)+ \\
& \quad+\underset{A, D}{\operatorname{Alt}} \operatorname{Tr}\left(\operatorname{Cycle}\left(D_{1} A_{1} \cdots D_{i-1} A_{i-1} \cdot A_{i} \cdot A_{i+1}\right) \cdot D_{i} A_{i+2}\right)
\end{aligned}
$$

The first summand in (7) cancels with the second one because of the symmetry and the main property of Tr; the third summand is

$$
\begin{aligned}
& i \cdot \operatorname{Alt} \operatorname{Tr}\left(D_{1} A_{1} \cdot \ldots \cdot D_{i} A_{i} \cdot A_{i+1} \cdot A_{i+2}\right)- \\
& \quad-\underset{A, D}{\operatorname{Alt}} \operatorname{Tr}\left(D_{1} A_{1} \cdot \ldots D_{i-1} A_{i-1} \cdot A_{i} \cdot D_{i} A_{i+1} \cdot A_{i+2}\right) .
\end{aligned}
$$

Furthermore let us consider an expression

$$
\underset{A, D}{\operatorname{Alt}} \operatorname{Tr}\left(D_{i}\left(D_{1} A_{1} \cdot \ldots \cdot D_{i-1} A_{i-1} \cdot A_{i} \cdot A_{i+1} \cdot A_{i+2}\right)\right) .
$$

It is clear that

$$
\begin{aligned}
(8)+(9)=(i+2) & \underset{A}{\operatorname{Alt}} \operatorname{Tr}\left(D_{1} A_{1} \cdot \ldots \cdot D_{i} A_{i} \cdot A_{i+1} \cdot A_{i+2}\right)= \\
& =(i+2) d\left(\operatorname{Alt}_{A, D}^{\operatorname{Alt}} \operatorname{Tr}\left(D_{1} A_{1} \cdot \ldots \cdot D_{i} A_{i} \cdot A_{i+1}\right)\right)\left(A_{i+2}, A_{1}, \ldots, A_{i+1}\right) .
\end{aligned}
$$

The terms which contain $\left[D_{a}, D_{b}\right]$ again cancel because $D_{1} \wedge \cdots \wedge D_{i}$ is a cycle.

2.3. We give here two examples of Lemma 2.2.

2.3.1.

Example. Let $\mathfrak{g}=\mathfrak{g l}_{n} \otimes \mathbb{C}\left[t_{1}, \ldots, t_{n}, t_{1}^{-1}, \ldots, t_{n}^{-1}\right]$. Define $\operatorname{Tr}: \mathfrak{g} \rightarrow \mathbb{C}$ to be the composition $\operatorname{Tr}=\operatorname{Res}_{t_{1}, \ldots, t_{n}} \circ \operatorname{Tr}_{\mathfrak{g l}_{n}}$. The associative algebra $\mathfrak{g}$ has $n$ exterior derivations: $D_{1}=\frac{d}{d t_{1}}, \ldots, D_{n}=\frac{d}{d t_{n}}$. Therefore, Lemma 2.2 gives the $(n+1)$-cocycle on the Lie algebra $\mathfrak{g}$. In particular, in the case $n=1$ we obtain the standard Kac-Moody 2-cocycle on the current algebra.

\subsection{2.}

Example. (i) Let $\mathfrak{A}$ be an associative algebra, $\mathfrak{h}$ an two-sided ideal in $\mathfrak{A}$. Then $\mathfrak{A}$ acts on the associate algebra $\mathfrak{h}$ via the adjoint action. Suppose, that the image of $\mathfrak{A}$ in $\operatorname{Der}_{\text {Ass }}(\mathfrak{h})$ lies in $\operatorname{Der}_{\text {Ass }}^{\operatorname{Tr}}(\mathfrak{h})$. Then Lemma 2.2 gives a map $q: H_{i}(\mathfrak{A} ; \mathbb{C}) \rightarrow H^{i+1}(\mathfrak{h} ; \mathbb{C})$.

(ii) In particular, let $\mathfrak{g l}_{\infty}^{\mathrm{Jac}}$ be an associative algebra of infinite matrices with a finite number (depending on the matrix) of nonzero diagonals, and let $\mathfrak{g} \mathfrak{l}_{\infty}^{\mathrm{fin}}$ be the ideal in $\mathfrak{g} \mathfrak{l}_{\infty}^{\mathrm{Jac}}$ consisting of the matrices with finite support. Then we have a map

$$
q: H_{i}\left(\mathfrak{g}_{\infty}^{\mathrm{Jac}} ; \mathbb{C}\right) \rightarrow H^{i+1}\left(\mathfrak{g l}_{\infty}^{\mathrm{fin}} ; \mathbb{C}\right) .
$$

Recall ([FT, Fu] $)$ that

$$
\begin{aligned}
H^{*}\left(\mathfrak{g l}_{\infty}^{\mathrm{Jac}} ; \mathbb{C}\right) & =S^{*}\left(c_{2}, c_{4}, c_{6}, \ldots\right) \\
H^{*}\left(\mathfrak{g l}_{\infty}^{\mathrm{fin}} ; \mathbb{C}\right) & =\Lambda^{*}\left(\xi_{1}, \xi_{3}, \xi_{5}, \ldots\right)
\end{aligned}
$$


This construction has an evident generalization: let $A$ be an associative algebra; then we have a map

$$
q: H_{*}\left(\mathfrak{g l}_{\infty}^{\mathrm{Jac}}(A) ; \mathbb{C}\right) \rightarrow H^{*+1}\left(\mathfrak{g l}_{\infty}^{\mathrm{fin}}(A) ; \mathbb{C}\right) .
$$

2.4. The case we have discussed in Sect. 2.2 is the simplest case of the lifting problem. The next step is to extend our construction to cycles in $H_{\operatorname{Tr}}^{1}(\mathfrak{A} ; \mathfrak{A})$. But we can do this only in the case $i=2$ (see $\S 1$ ). In the cases $i=4,6,8, \ldots$ the most we can hope is to solve the problem with some additional conditions. We thus assume that the following conditions (i)-(iii) hold:

(i) $D_{j} \in \operatorname{Der}_{\text {Ass }}^{\operatorname{Tr}}(\mathfrak{A})$ for all $j$;

(ii) we solve problem only for one monomial $D_{1} \wedge \cdots \wedge D_{i} \quad(k=1)$, and

$$
\left[D_{a}, D_{b}\right]=\operatorname{ad} Q_{a b} \text { - inner derivations }\left(Q_{a b} \in \mathfrak{A}\right) \text {; }
$$

(iii) $\operatorname{Alt}_{a, b, c} D_{c}\left(Q_{a b}\right)=0$ for all $a, b, c$.

Main Conjecture (First Version). Under the conditions (i)-(iii) the lifting problem can be solved for $\alpha_{D_{1}, \ldots, D_{i}}^{*}$.

We expect that

$$
\begin{aligned}
\Psi_{i+1}\left(A_{1}, \ldots, A_{i+1}\right)= & \operatorname{Alt}_{A, D} \operatorname{Tr}\left(D_{1} A_{1} \cdot \ldots \cdot D_{i} A_{i} \cdot A_{i+1}\right)+ \\
& +\left(\text { terms, linear in } Q_{a b}\right)+\left(\text { terms, quadratic in } Q_{a b}\right)+\ldots
\end{aligned}
$$

(by analogy with Theorem 1.1).

Remark. It follows easily from the Jacobi identity that $\underset{a, b, c}{\operatorname{Alt}} D_{c}\left(Q_{a b}\right)$ lies in the center $Z$ of Lie algebra $\mathfrak{A}$. Hence, codition (iii) is not very strong; in particular, it holds for the Lie factoralgebra $\mathfrak{A} / Z$.

Here the Conjecture will be proved only for $n=1,2$; but we will conject in Sect. 4.6 an explicit formula for all $n$.

The lifting formula for $n=2$ contains terms quadratic in $Q_{a b}$.

2.5. The main example when conditions (i)-(iii) hold is an associative algebra $\Psi \operatorname{Dif}_{n}\left(S^{1}\right)$ of pseudo-differential operators on $\left(S^{1}\right)^{n}$ and $2 \cdot n$ exterior derivations of $\Psi \operatorname{Dif}_{n}\left(S^{1}\right): \ln x_{1}, \ldots, \ln x_{n}, \ln \partial_{1}, \ldots, \ln \partial_{n}$. In fact, we can define derivation $\ln \mathcal{D}$ (for $\left.\mathcal{D} \in \Psi \operatorname{Dif}_{n}\left(S^{1}\right)\right)$ in much more generality. It seems to be true that to do this we need only this condition:

There exists $\mathcal{D}^{*} \in \Psi \operatorname{Dif}_{n}\left(S^{1}\right)$ such that $\left[\mathcal{D}, \mathcal{D}^{*}\right]=1$.

But, first, we have to remember about conditions (i)-(iii), and, second, we will see in Sect. 2.8 that in fact the choice $\left\{\ln x_{1}, \ldots, \ln x_{n} ; \ln \partial_{1}, \ldots, \ln \partial_{n}\right\}$ is, in the some sense, most general.

2.6. There is a deformation of the Lie algebra $\operatorname{Poiss}_{2 n}\left(S^{1}\right)$ to the Lie algebra $\Psi \operatorname{Dif}_{n}\left(S^{1}\right)$. If we suppose that that the lifting problem can be solved, it is interesting to find the Hamiltonian limit of the lifting formulas. It is easy to see that in (10), this limit depends only on the leading term, but not on the terms containing $Q_{a b}$. Let us describe this cocycles.

Let $p_{1}, \ldots, p_{n} ; q_{1}, \ldots, q_{n}$ be standard Poisson coordinates, $\left\{p_{i}, q_{j}\right\}=\delta_{i j}$. 
Let $\operatorname{Tr}: \operatorname{Poiss}_{2 n}\left(S^{1}\right) \rightarrow \mathbb{C}$ be the linear functional $\operatorname{Tr}=\operatorname{Res}_{p_{1}} \circ \ldots \circ \operatorname{Res}_{p_{n}} \circ \operatorname{Res}_{q_{1}} \circ \ldots \circ$ $\operatorname{Res}_{q_{n}}$.

Lemma. $\operatorname{Tr}\{f, g\}=0$ for any $f, g \in \operatorname{Poiss}_{2 n}\left(S^{1}\right)$.

Proof.

$$
\{f, g\}=\sum_{k}\left(\frac{\partial f}{\partial p_{k}} \frac{\partial g}{\partial q_{k}}-\frac{\partial f}{\partial q_{k}} \frac{\partial g}{\partial p_{k}}\right)=\sum_{k}\left(\frac{\partial}{\partial p_{k}}\left(f \cdot \frac{\partial g}{\partial q_{k}}\right)-\frac{\partial}{\partial q_{k}}\left(f \cdot \frac{\partial g}{\partial p_{k}}\right)\right) .
$$

2.7. Any $\mathcal{D} \in \operatorname{Poiss}_{2 n}\left(S^{1}\right), \mathcal{D} \neq 0$, determines an exterior derivation $\ln \mathcal{D}: \operatorname{Poiss}_{2 n}\left(S^{1}\right) \rightarrow \operatorname{Poiss}_{2 n}\left(S^{1}\right)$ by the formula

$$
\{\ln \mathcal{D}, f\}=\frac{\{\mathcal{D}, f\}}{\mathcal{D}}
$$

Lemma. For any $\mathcal{D}_{1}, \ldots, \mathcal{D}_{2 n} \in \operatorname{Poiss}_{2 n}\left(S^{1}\right)$ the formula

$$
\Psi_{2 n+1}^{\mathcal{D}_{1}, \ldots, \mathcal{D}_{n}}\left(f_{1}, \ldots, f_{2 n+1}\right)=\underset{f}{\operatorname{Alt}} \operatorname{Tr}\left(\left\{\ln \mathcal{D}_{1}, f_{1}\right\} \cdot \ldots \cdot\left\{\ln \mathcal{D}_{2 n}, f_{2 n}\right\} \cdot f_{2 n+1}\right)
$$

defines a $(2 n+1)$-cocycle on $\operatorname{Poiss}_{2 n}\left(S^{1}\right)$.

Proof. We will proof, that

$$
\Psi_{2 n+1}^{F}\left(f_{1}, \ldots, f_{2 n+1}\right)=\operatorname{Alt}_{f} \operatorname{Tr}\left(F \cdot \frac{\partial f_{1}}{\partial p_{1}} \cdot \ldots \cdot \frac{\partial f_{n}}{\partial p_{n}} \cdot \frac{\partial f_{n+1}}{\partial q_{1}} \cdot \ldots \cdot \frac{\partial f_{2 n}}{\partial q_{n}} \cdot f_{2 n+1}\right)
$$

defines a $(2 n+1)$-cocycle on $\operatorname{Poiss}_{2 n}\left(S^{1}\right)$. It is easy to see, that

$$
\Psi_{2 n+1}^{\mathcal{D}_{1}, \ldots, \mathcal{D}_{2 n}}=\Psi_{2 n+1}^{F}
$$

for $F=\frac{\operatorname{det}\left(\frac{\partial \mathcal{D}_{i}}{\partial \xi_{j}}\right)}{\mathcal{D}_{1} \cdot \ldots \cdot \mathcal{D}_{2 n}} \quad\left(\right.$ here $\left.\xi_{1}=p_{n}, \ldots, \xi_{n}=p_{n}, \xi_{n+1}=q_{1}, \ldots, \xi_{2 n}=q_{n}\right)$.

We have:

$$
\begin{array}{r}
(12) \quad\left(d \Psi_{2 n+1}^{F}\right)\left(f_{2 n+2}, f_{1}, \ldots, f_{2 n+1}\right)= \\
=\frac{1}{2} \operatorname{Alt}_{f} \operatorname{Tr}\left[F \cdot \frac{\partial}{\partial p_{1}}\left\{f_{2 n+2} f_{1}\right\} \cdot \frac{\partial}{\partial p_{2}} f_{2} \cdot \frac{\partial}{\partial p_{3}} f_{3} \cdot \ldots+F \cdot \frac{\partial}{\partial p_{1}} f_{1} \cdot \frac{\partial}{\partial p_{2}}\left\{f_{2 n+2}, f_{2}\right\} \cdot \frac{\partial}{\partial p_{3}} f_{3} .\right. \\
\left.\ldots+\ldots++F \cdot \frac{\partial}{\partial p_{1}} f_{1} \cdot \ldots \cdot \frac{\partial}{\partial q_{n}} f_{2 n} \cdot\left\{f_{2 n+2}, f_{2 n+1}\right\}\right] .
\end{array}
$$

We subtract from the r.h.s. of (12) the expression

$$
\frac{1}{2} \operatorname{Alt} \operatorname{Tr}\left(\left\{f_{2 n+2}, F \cdot \frac{\partial}{\partial p_{1}} f_{1} \cdot \ldots \cdot \frac{\partial}{\partial q_{n}} f_{2 n} \cdot f_{2 n+1}\right\}\right),
$$

which is equal to zero by Lemma 2.6.

We have:

$$
\begin{aligned}
& \text { (13) r.h.s. }(12)=-\frac{1}{2} \underset{f}{\operatorname{Alt}} \operatorname{Tr}\left(\left\{f_{2 n+2}, F\right\} \cdot \frac{\partial}{\partial p_{1}} f_{1} \cdot \ldots \cdot \frac{\partial}{\partial q_{n}} f_{2 n} \cdot f_{2 n+1}\right) \\
& +\frac{1}{2} \operatorname{Alt} \operatorname{Tr}\left[F \cdot\left\{\frac{\partial}{\partial p_{1}} f_{2 n+2}, f_{1}\right\} \cdot \frac{\partial}{\partial p_{2}} f_{2} \cdot \ldots+F \cdot \frac{\partial}{\partial p_{1}} f_{1} \cdot \frac{\partial}{\partial p_{2}}\left\{f_{2 n+2}, f_{2}\right\} \cdot \ldots+\ldots\right] .
\end{aligned}
$$


The second summand is equal to the first one by Lemma 2.5 and the symmetry, and we have:

$$
\left(d \Psi_{2 n+1}^{F}\right)\left(f_{2 n+2}, f_{1}, \ldots, f_{2 n+1}\right)=\underset{f}{\operatorname{Alt}} \operatorname{Tr}\left(\left\{F, f_{2 n+2}\right\} \cdot \frac{\partial}{\partial p_{1}} f_{1} \cdot \ldots \frac{\partial}{\partial q_{n}} f_{2 n} \cdot f_{2 n+1}\right) .
$$

Furthermore $\left\{F, f_{2 n+2}\right\}=\sum_{k}\left(\frac{\partial F}{\partial p_{k}} \frac{\partial f_{2 n+2}}{\partial q_{k}}-\frac{\partial F}{\partial q_{k}} \cdot \frac{\partial f_{2 n+2}}{\partial p_{k}}\right)$ and every summand in (14) is equal to zero via the alternation.

2.8. Although Lemma 2.7 gives us a lot of cocycles, it is easy to see that $\Psi_{2 n+1}^{F_{1}} \sim$ $\Psi_{2 n+1}^{F_{2}}$ when Res $F_{1}=\operatorname{Res} F_{2}$; therefore, we have only one interesting cocycle, which is $\Psi_{2 n+1}^{\ln p_{1}, \ldots, \ln p_{n}, \ln q_{1}, \ldots, \ln q_{n}}$. We will denote it by $\Psi_{2 n+1}^{0}$.

Lemma. (i) If the lifting problem for $\Psi \operatorname{Dif}_{n}\left(S^{1}\right)$ can be solved, the Hamiltonian limit of any lifting is $\Psi_{2 n+1}^{0}$;

(ii) $\Psi_{2 n+1}^{0}\left(1 \wedge p_{1} \wedge \cdots \wedge p_{n} \wedge q_{1} \wedge \cdots \wedge q_{n}\right) \neq 0$.

This Lemma implies that the lifting problem $=$ the deformation problem.

On the other hand, we see that we don't need try to generalize the construction for $\Psi \operatorname{Dif}_{n}\left(S^{1}\right)$ to other derivations of the form $\ln \mathcal{D}$; the case $D_{1}=\ln x_{1}, \ldots, D_{2 n}=\ln \partial_{n}$ is most general.

Corollary. Any $(2 n+1)$-cocycle on the Lie algebra $\Psi \operatorname{Dif}_{n}\left(S^{1}\right)$ of the form $(10)\left(D_{1}=\right.$ $\left.\ln x_{1}, \ldots, D_{2 n}=\ln \partial_{n}\right)$ is not cohomologous to zero.

Another proof follows from the fact that every cocycle of the form (10) is a lifting of $\alpha_{\ln x_{1}, \ldots, \ln \partial_{n}}^{*}$, which is a nonzero element in $H^{2 n}\left(\Psi \operatorname{Dif}_{n}\left(S^{1}\right) ; \Psi \operatorname{Dif}_{n}\left(S^{1}\right)^{*}\right)$.

\section{Computation for $n=2$}

3.1. The main result of this Section is the following

Theorem. Let $\mathfrak{A}$ be an associative algebra, $\operatorname{Tr}: \mathfrak{A} \rightarrow \mathbb{C}$ be a trace on $\mathfrak{A}$ and $D_{1}, D_{2}, D_{3}$, $D_{4}$ - four (exterior) derivations on $\mathfrak{A}$, which satisfy conditions (i)-(iii) from Sect. 2.4. Then

$$
\begin{aligned}
& \Psi_{5}\left(A_{1}, A_{2}, A_{3}, A_{4}, A_{5}\right)=\operatorname{Alt}_{A, D}\left\{D_{1} A_{1} \cdot D_{2} A_{2} \cdot D_{3} A_{3} \cdot D_{4} A_{4} \cdot A_{5}\right. \\
& +A_{1} Q_{12} A_{2} \cdot D_{3} A_{3} \cdot D_{4} A_{4} \cdot A_{5} \\
& +D_{1} A_{1} \cdot A_{2} \cdot Q_{23} \cdot A_{3} \cdot D_{4} A_{4} \cdot A_{5} \quad \text { terms, linear in } Q_{i j} \\
& +D_{1} A_{1} \cdot D_{2} A_{2} \cdot A_{3} \cdot Q_{34} \cdot A_{4} \cdot A_{5} \\
& \left.\left.+A_{1} Q_{12} A_{2} A_{3} Q_{34} A_{4} A_{5}\right\}\right] \text { term, quadric in } Q_{i j}
\end{aligned}
$$

is a 5-cocycle on the Lie algebra $\mathfrak{A}$, which is the lifting of $\alpha_{D_{1}, D_{2}, D_{3}, D_{4}}^{*}$ (see Sect. 2.1). In the case $\mathfrak{A}=\Psi \operatorname{Dif}_{2}\left(S^{1}\right), D_{1}=\ln \partial_{1}, D_{2}=\ln x_{1}, D_{3}=\ln \partial_{2}, D_{4}=\ln x_{2}, \Psi_{5}$ is not cohomologous to zero: $\Psi_{5}\left(1 \wedge x_{1} \wedge x_{2} \wedge \partial_{1} \wedge \partial_{2}\right) \neq 0$.

Remark. ad $Q_{i j}=\left[D_{i}, D_{j}\right]$; however, in formula (15), we don't alternate symbols $i$ and $j$ in $Q_{i j}$. 
We will give a sketch of the proof of this Theorem in Sect. 3.2-3.5.

3.2 .

\section{Lemma.}

(i) $d\left(\operatorname{Alt}_{A, D} \operatorname{Tr}\left(D_{1} A_{1} \cdot A_{2} \cdot A_{3} \cdot D_{2} A_{4} \cdot Q \cdot A_{5}\right)\left(A_{6}, A_{1}, \ldots, A_{5}\right)=\right.$

$$
=\underset{A, D}{\operatorname{Alt}} \operatorname{Tr}\left(D_{1} A_{1} \cdot A_{2} \cdot A_{3} \cdot D_{2} A_{6} \cdot A_{4} \cdot Q \cdot A_{5}\right)
$$

(ii) $d\left(\underset{A, D}{\operatorname{Alt}} \operatorname{Tr}\left(D_{1} A_{1} \cdot D_{2} A_{2} \cdot A_{3} \cdot A_{4} \cdot Q \cdot A_{5}\right)\left(A_{6}, A_{1}, \ldots, A_{5}\right)=\right.$

$$
=\operatorname{Alt}_{A, D} \operatorname{Tr}\left(D_{1} A_{1} \cdot D_{2} A_{6} \cdot A_{2} \cdot A_{3} \cdot A_{4} \cdot Q \cdot A_{5}\right)
$$

(iii) $d\left(\operatorname{Alt}_{A, D} \operatorname{Tr}\left(D_{1} A_{1} \cdot D_{2} A_{2} \cdot A_{3} \cdot A_{4} \cdot Q \cdot A_{5}\right)\left(A_{6}, A_{1}, \ldots, A_{5}\right)=\right.$

$$
=\underset{A, D}{\operatorname{Alt}} \operatorname{Tr}\left(D_{1} A_{1} \cdot D_{2} A_{2} \cdot A_{3} \cdot Q \cdot A_{6} \cdot A_{4} \cdot A_{5}\right)
$$

Proof. Straightforward (see Sect. 1.3).

\section{3 .}

\section{Lemma.}

(i) $d\left(\operatorname{Alt}_{A, D} \operatorname{Tr}\left(D_{1} A_{1} \cdot A_{2} \cdot A_{3} \cdot A_{4} \cdot D_{2} A_{5} \cdot Q\right)\right)\left(A_{6}, A_{1}, \ldots, A_{5}\right)=$

$$
=\underset{A, D}{\operatorname{Alt}} \operatorname{Tr}\left(D_{1} A_{6} \cdot A_{1} \cdot A_{2} \cdot A_{3} \cdot A_{4} \cdot D_{2} A_{5} \cdot Q-D_{1} A_{1} \cdot A_{2} \cdot A_{3} \cdot A_{4} \cdot D_{2} A_{5} \cdot\left[A_{6}, Q\right]\right)
$$

(ii) $d\left(\operatorname{Alt}_{A, D} \operatorname{Tr}\left(D_{1} A_{1} \cdot A_{2} \cdot D_{2} A_{3} \cdot A_{4} \cdot A_{5} \cdot Q\right)\right)\left(A_{6}, A_{1}, \ldots, A_{5}\right)=$

$$
=\operatorname{Alt}_{A, D} \operatorname{Tr}\left(D_{1} A_{6} \cdot A_{1} \cdot A_{2} \cdot D_{2} A_{3} \cdot A_{4} \cdot A_{5} \cdot Q-D_{1} A_{1} \cdot A_{2} \cdot D_{2} A_{3} \cdot A_{4} \cdot A_{5} \cdot\left[A_{6}, Q\right]\right)
$$

(iii) $d\left(\operatorname{Alt}_{A, D} \operatorname{Tr}\left(D_{1} A_{1} \cdot A_{2} \cdot D_{2} A_{3} \cdot Q \cdot A_{4} \cdot A_{5}\right)\right)\left(A_{6}, A_{1}, \ldots, A_{5}\right)=$

$$
=\underset{A, D}{\operatorname{Alt}} \operatorname{Tr}\left(D_{1} A_{6} \cdot A_{1} \cdot A_{2} \cdot D_{2} A_{3} \cdot Q \cdot A_{4} \cdot A_{5}-D_{1} A_{1} \cdot A_{2} \cdot D_{2} A_{3} \cdot\left[A_{6}, Q\right] \cdot A_{4} \cdot A_{5}\right)
$$


3.4 .

\section{Lemma.}

(i) $d\left(\underset{A, D}{\operatorname{Alt}} \operatorname{Tr}\left(D_{1} A_{1} \cdot D_{2} A_{2} \cdot D_{3} A_{3} \cdot D_{4} A_{4} \cdot A_{5}\right)\right)\left(A_{6}, A_{1}, \ldots, A_{5}\right)=$

$$
=\underset{A, D}{\operatorname{Alt}} \operatorname{Tr}\left(D_{1} A_{1} \cdot D_{2} A_{2} \cdot D_{3} A_{3} \cdot D_{4} A_{4} \cdot A_{5} \cdot A_{6}\right)
$$

(ii) $d\left(\operatorname{Alt}_{A, D} \operatorname{Tr}\left(Q_{1} \cdot Q_{2} \cdot A_{1} \cdot A_{2} \cdot A_{3} \cdot A_{4} \cdot A_{5}\right)\right)\left(A_{6}, A_{1}, \ldots, A_{5}\right)=$

$$
=-\operatorname{Alt}_{A} \operatorname{Tr}\left(Q_{1} \cdot Q_{2} \cdot A_{1} \cdot A_{2} \cdot A_{3} \cdot A_{4} \cdot A_{5} \cdot A_{6}\right)
$$

(iii) $d\left(\operatorname{Alt}_{A, D} \operatorname{Tr}\left(Q_{1} \cdot A_{1} \cdot Q_{2} \cdot A_{2} \cdot A_{3} \cdot A_{4} \cdot A_{5}\right)\right)\left(A_{6}, A_{1}, \ldots, A_{5}\right)=$

$$
=\operatorname{Alt}_{A} \operatorname{Tr}\left(Q_{1} \cdot A_{6} \cdot A_{1} \cdot Q_{2} \cdot A_{2} \cdot A_{3} \cdot A_{4} \cdot A_{5}\right)
$$

3.5. To prove Theorem 3.1, we consider an expression in $A_{1}, \ldots, A_{6}$ which a priori is equal to zero (we use $\operatorname{Tr}(D A)=0$ - condition (i) from Sect. 2.4), and then prove that this expression is a coboundary of $\Psi_{5}\left(A_{1}, \ldots, A_{5}\right)$. Then $\Psi_{5}$ is a 5-cocycle. Our main tools are Lemmas 3.2-3.4.

Suppose that

$$
\begin{gathered}
E_{1}=\operatorname{Alt}_{A, D} \operatorname{Tr}\left(2 D_{2}\left(Q_{43} \cdot D_{1} A_{1} \cdot A_{2} \cdot A_{3} \cdot A_{4} \cdot A_{5} \cdot A_{6}\right)+\right. \\
\left.+2 D_{2}\left(D_{1} A_{1} \cdot Q_{43} \cdot A_{2} \cdot A_{3} \cdot A_{4} \cdot A_{5} \cdot A_{6}\right)\right) \\
E_{2}=\operatorname{Alt}_{A, D} \operatorname{Tr}\left(D_{2}\left(D_{1} A_{1} \cdot A_{2} \cdot A_{3} \cdot Q_{43} \cdot A_{4} \cdot A_{5} \cdot A_{6}\right)+\right. \\
\left.+D_{2}\left(D_{1} A_{1} \cdot A_{2} \cdot A_{3} \cdot A_{4} \cdot Q_{43} \cdot A_{5} \cdot A_{6}\right)\right) \\
E_{3}=\operatorname{Alt}_{A, D} \operatorname{Tr}\left(D_{4}\left(D_{1} A_{1} \cdot A_{2} \cdot A_{3} \cdot A_{4} \cdot Q_{43} \cdot A_{5} \cdot A_{6}\right)\right)
\end{gathered}
$$

Note that $E_{1}=E_{2}=E_{3}=0$.

Furthermore suppose that

$$
\begin{gathered}
I=\operatorname{Alt}_{A, D} \operatorname{Tr}\left(D_{2}\left(\left(D_{1} A_{1} \cdot A_{2} \cdot A_{3} \cdot A_{4}\right) \cdot D_{4}\left(A_{6} \cdot D_{3} A_{5}\right)\right)\right) \\
I I=\operatorname{Alt}_{A, D} \operatorname{Tr}\left(D_{2}\left(\left(A_{3} \cdot A_{4} \cdot D_{1} A_{1} \cdot A_{2}\right) \cdot D_{4}\left(A_{6} \cdot D_{3} A_{5}\right)\right)\right) \\
I I I=\operatorname{Alt}_{A, D} \operatorname{Tr}\left(D_{2}\left(\left(A_{2} \cdot A_{3} \cdot A_{4} \cdot D_{1} A_{1}\right) \cdot D_{4}\left(D_{3} A_{5} \cdot A_{6}\right)\right)\right) \\
I V=\operatorname{Alt}_{A, D} \operatorname{Tr}\left(D_{2}\left(\left(A_{4} \cdot D_{1} A_{1} \cdot A_{2} \cdot A_{3}\right) \cdot D_{4}\left(D_{3} A_{5} \cdot A_{6}\right)\right)\right)
\end{gathered}
$$

Note also that $I=I I=I I I=I V=0$. 
We state that

$$
E_{1}-E_{2}+\frac{4}{3} E_{3}-I+I I-I I I+I V=d \widetilde{\Psi}_{5}
$$

where

$$
\begin{aligned}
& \widetilde{\Psi}_{5}=\operatorname{Alt}_{A, D} \operatorname{Tr}\left(-2 D_{1} A_{1} \cdot D_{2} A \cdot D_{3} A_{3} \cdot D_{4} A_{4} \cdot A_{5}\right] \text { term without } Q_{i j} \\
& +D_{1} A_{1} \cdot D_{2} A_{2} \cdot Q_{43} \cdot A_{3} \cdot A_{4} \cdot A_{5} \\
& +D_{1} A_{1} \cdot D_{2} A_{2} \cdot A_{3} \cdot A_{4} \cdot A_{5} \cdot Q_{43} \\
& +2 \cdot D_{1} A_{1} \cdot Q_{43} \cdot D_{2} A_{2} \cdot A_{3} \cdot A_{4} \cdot A_{5} \quad \text { terms, linear in } Q_{i j} \\
& -D_{1} A_{1} \cdot A_{2} \cdot D_{2} A_{3} \cdot Q_{43} \cdot A_{4} \cdot A_{5} \\
& -D_{1} A_{1} \cdot A_{2} \cdot D_{2} A_{3} \cdot A_{4} \cdot A_{5} \cdot Q_{43} \\
& \left.-4 Q_{21} \cdot Q_{43} \cdot A_{1} \cdot A_{2} \cdot A_{3} \cdot A_{4} \cdot A_{5}\right] \\
& +2 Q_{21} \cdot A_{1} \cdot A_{2} \cdot Q_{43} \cdot A_{3} \cdot A_{4} \cdot A_{5} \quad \text { terms, quadratic in } Q_{i j} \\
& \left.-2\left[Q_{21}, A_{1}\right] \cdot\left[Q_{43}, A_{2}\right] \cdot A_{3} \cdot A_{4} \cdot A_{5}\right]
\end{aligned}
$$

To prove this, we need condition (iii) from Sect. 2.4:

$$
\underset{i, j, k}{\operatorname{Alt}} D_{k}\left(Q_{i j}\right)=0 \quad \text { for all } i, j, k \text {. }
$$

The proof of (16) is a very long straightforward calculation, using Lemmas 3.2-3.4 and other observations.

Furthermore, after simple manipulations

$\widetilde{\Psi}_{5}-\operatorname{Alt}_{A, D} \operatorname{Tr}\left(D_{2}\left(D_{1} A_{1} \cdot Q_{43} \cdot A_{2} \cdot A_{3} \cdot A_{4} \cdot A_{5}\right)\right)-\operatorname{Alt}_{A, D} \operatorname{Tr}\left(D_{1}\left(A_{1} \cdot Q_{43} \cdot D_{2} A_{2} \cdot A_{3} \cdot A_{4} \cdot A_{5}\right)\right)$

will have a form as in Th. 3.1.

\section{Lifting FORMUlas: the General CASE}

4.1. In this Section the lifting formulas for any number of derivations $D_{1}, \ldots, D_{l}$ (with conditions (i)-(iii) from Sect. 2.4) appear. The fact that polylinear skew-symmetric functions on $\mathfrak{A}$, defined by these formulas, are cocycles, is the Main Conjecture of this Section. As we have seen in $\S 3$, in our situation it is highly nontrivial to check that a given formula in fact defines a cocycle.

The main idea is the following. We consider the expression

$$
\operatorname{Alt}_{A, D} \operatorname{Tr}\left(D_{1} A_{1} \cdot D_{2} A_{2} \cdots D_{2 n} A_{2 n} \cdot A_{2 n+1}\right)
$$

where $D_{1}, \ldots, D_{2 n}$ are inner derivations, $D_{i} A=D_{i} \cdot A-A \cdot D_{i}$. We want to add to (17) some terms containing $Q_{i j}=\left[D_{i}, D_{j}\right]$ and in this way obtain a cocycle. It is meaningless from the cohomological viewpoint, because this cocycle will be cohomologous to 0 (see Remark 4.2 and Remark 4.5), but using this trick we obtain explicit formulas.

We have:

$(17)=\operatorname{Alt}_{A, D} \operatorname{Tr}\left(\left(D_{1} \cdot A_{1}-A_{1} \cdot D_{1}\right) \cdot\left(A_{2} \cdot D_{2}-D_{2} \cdot A_{2}\right) \cdot \ldots \cdot\left(D_{2 n} \cdot A_{2 n}-A_{2 n} \cdot D_{2 n}\right) \cdot A_{2 n+1}\right)$

Note also that our formulas are true for any number of derivations, not only for an even number. 
4.2 .

Lemma. Let $\mathfrak{A}$ be an associative algebra; $D_{1}, \ldots, D_{2 n} \in \mathfrak{A}$. Then

$$
\alpha\left(A_{1}, \ldots, A_{2 n+1}\right)=\operatorname{Alt}_{A, D} \operatorname{Tr}\left(D_{1} \cdot A_{1} \cdot D_{2} \cdot A_{2} \cdot \ldots \cdot D_{2 n} \cdot A_{2 n} \cdot A_{2 n+1}\right)
$$

is a $(2 n+1)$-cocycle on the Lie algebra $\mathfrak{A}$.

Proof.

$$
\begin{aligned}
& d \alpha\left(A_{2 n+2}, A_{1}, \ldots, A_{2 n+1}\right)= \\
& =\frac{1}{4} \operatorname{Alt}_{A, D} \operatorname{Tr}\left\{D_{1} \cdot\left[A_{2 n+2}, A_{1}\right] \cdot D_{2} \cdot A_{2} \cdot \ldots \cdot D_{2 n} \cdot\left[A_{2 n}, A_{2 n+1}\right]\right. \\
& \quad+D_{1} \cdot A_{1} \cdot D_{2} \cdot\left[A_{2 n+2}, A_{2}\right] \cdot D_{3} \cdot A_{3} \cdot \ldots \cdot D_{2 n} \cdot\left[A_{2 n}, A_{2 n+1}\right] \\
& \quad+\ldots \\
& \quad+D_{1} \cdot A_{1} \cdot D_{2} \cdot A_{2} \cdot \ldots \cdot D_{2 n-1} \cdot\left[A_{2 n+2}, A_{2 n-1}\right] \cdot D_{2 n} \cdot\left[A_{2 n}, A_{2 n+1}\right] \\
& \quad+D_{1} \cdot A_{1} \cdot \ldots \cdot D_{2 n} \cdot\left[\left[A_{2 n+2},\left[A_{2 n}, A_{2 n+1}\right]\right]\right\} .
\end{aligned}
$$

The last summand in (18) is equal to 0 by the Jacobi identity. The remaining summands cancel by the trace property and symmetry conditions. For example,

$$
\begin{aligned}
\operatorname{Alt}_{A, D} \operatorname{Tr}\left\{D _ { 1 } \cdot \left[A_{2 n+2},\right.\right. & \left.A_{1}\right] \cdot D_{2} \cdot A_{2} \cdot \ldots \cdot D_{2 n} \cdot\left[A_{2 n}, A_{2 n+1}\right]+ \\
& \left.+D_{1} \cdot A_{1} \cdot \ldots \cdot D_{2 n-1} \cdot\left[A_{2 n+2}, A_{2 n-1}\right] \cdot D_{2 n} \cdot\left[A_{2 n}, A_{2 n+1}\right]\right\}=0 .
\end{aligned}
$$

Note also that the proof remains the same in the case when there are an odd number of $D_{i}$.

Remark. Indeed, $\alpha$ is a coboundary, $\alpha=a \cdot d\left(D_{1} \cdot A_{1} \cdot \ldots \cdot D_{2 n} \cdot A_{2 n}\right) \quad(a \in \mathbb{Z})$.

4.3. Let $D_{i} \in \mathfrak{A}$ for all $i$. Then

$$
\operatorname{Alt}_{A, D} \operatorname{Tr}\left(\left[D_{1}, A_{1}\right] \cdot \ldots \cdot\left[D_{2 n}, A_{2 n}\right] \cdot A_{2 n+1}\right)=k \alpha+S \quad(k \in \mathbb{Z})
$$

where

$$
S=\left(\text { sum of the terms which contains } D_{i} \cdot D_{i+1} \text { for some } i\right)
$$

We replace in the all summands of $S D_{i} D_{i+1}$ by $\left[D_{i}, D_{i+1}\right]$ because of the symmetry condition. Hence, these terms have the same form as the terms in the lifting formulas, and our problem is to represent $S$ as a sum of the terms of the form

$$
\begin{aligned}
\operatorname{Alt}_{A, D} \operatorname{Tr}\left(\left[D_{1}, A_{1}\right] \cdot \ldots \cdot A_{i_{1}} \cdot Q_{i_{1}, i_{1}+1} A_{i_{1}+1} \cdot\left[D_{i_{1}+2}, A_{i_{1}+2}\right]\right. & \\
& \left.\ldots \cdot A_{i_{2}} \cdot Q_{i_{2}, i_{2}+1} \cdot A_{i_{2}+1} \cdot\left[D_{i_{2}+2}, A_{i_{2}+2}\right] \cdot \ldots\right) .
\end{aligned}
$$

Then, when we subtract $S$ from (19) we obtain $k \alpha$, which is a cocycle by Lemma 2.1. Indeed, in this case $\left(D_{i} \in A\right), k \alpha$ is a coboundary. But it turns out that when the $D_{i}$ are exterior derivations satisfying conditions (i)-(iii) from Sect. 2.4 and $n=1,2$, the lifting formulas from $\S 1,3$ have the same form. This fact allows us to formulate the Main Conjecture in Sect. 4.6. 
4.4. We will denote summands from $S$ by closed intervals with marked points. If $2 n$ is the total number of $D_{i}$, the length of the interval is $2 n-2$, and some integral points on it are marked. Let us denote by $1, \ldots, 2 n-1$ the integral points of the interval; the point $i$ is marked, if in the corresponding summand in $S, D_{i}$ and $D_{i+1}$ are neighbors (i.e. not separated by $A_{i}$ or $A_{i+1}$, or $A_{i} \cdot A_{i+1}$ ). For example, the interval

corresponds to the expression

$$
\operatorname{Alt}_{A, D} \operatorname{Tr}\left(A_{1} \cdot D_{1} \cdot D_{2} \cdot A_{2} \cdot\left[D_{3}, A_{3}\right] \cdot\left[D_{4}, A_{4}\right] \cdot A_{5}\right) .
$$

It is clear that the distance between the marked points is $\geq 2$. In the general case, the interval

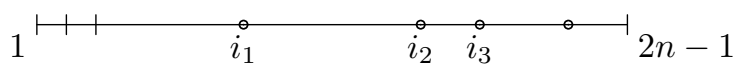

corresponds to the expression

$$
\begin{array}{r}
\operatorname{Alt}_{A, D} \operatorname{Tr}\left(\left[D_{1}, A_{1}\right] \cdot\left[D_{2}, A_{2}\right] \cdot \ldots \cdot A_{i_{1}} \cdot D_{i_{1}} \cdot D_{i_{1}+1} \cdot A_{i_{1}+1} \cdot\left[D_{i_{1}+2}, A_{i_{1}+2}\right]\right. \\
\\
\left.\cdot \ldots \cdot A_{i_{2}} \cdot D_{i_{2}} \cdot D_{i_{2}+1} A_{i_{2}+1} \cdot \ldots\right)
\end{array}
$$

Let $N$ be the total number of the marked points, $1 \leq N \leq n$.

The first summand in $S$ is a sum of all intervals with $N=1$, with sign "-" $(2 n-1$ intervals):

$$
S_{1}=-\left(\begin{array}{c:ccc}
0 & 1 & 1 \\
\hdashline & 1 & 1 \\
\hdashline & 0 & 1 \\
\hdashline & & \vdots \\
\hdashline & 1 & 1 & \vdots
\end{array}\right)
$$

FIGURE $1 . n=3$

Some summands of $S$ are counted in $S_{1}$ with multiplicities $>1$. We subtract from $S_{1}$ all summands with $N=2$ pairs of $D_{i}$, and then we add every interval with $N=2$ marked points with its sign in $S$. We have:

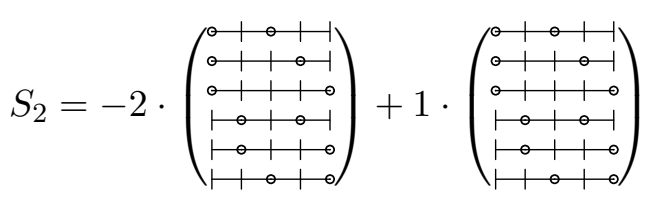

Figure 2. $S_{2}$ for $n=3$

We wrote the coefficient -2 because every interval with $N=2$ marked points is "contained" in two intervals with $N=1$ marked points with the sign "_"; for example, the interval $\bullet+\bullet+1$ "contains" in $\bullet 1+1+1$ and in $\mapsto \bullet \cdot 1$. In the sum $S_{1}+S_{2}$ only the terms with $N \geq 3$ pairs of $D_{i}$ are counted incorrectly. In our example $(n=3)$ the maximal value for $N$ is $N=3$, and there exists just one unique interval with $N=3: \bullet 1 \bullet 1 \bullet$. 
It contains with the sign " + " in 3 intervals with $N=1$ marked points, namely, in

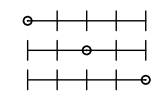

FiguRE 3.

and with the sign "-" in 3 intervals with $N=2$ marked points:

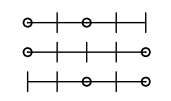

FiguRE 4.

Hence,

$$
S_{3}=3 \cdot \bullet 1 \cdot 1 \cdot-3 \cdot \bullet|\cdot| \cdot-1 \cdot \bullet 1 \cdot 1 \cdot=-1 \cdot \bullet|\cdot| \cdot \mid
$$

Note that all coefficients in $S_{1}, S_{2}, S_{3}$ are equal to -1 .

We have: $S=S_{1}+S_{2}+S_{3}$.

4.5. Let us denote by $\Sigma_{N, 2 n-1}$ the sum of all intervals of length $2 n-2$ with $N$ marked points $(1 \leq N \leq n$, the distance between marked points is $\geq 2)$.

\section{Lemma.}

$$
S=-\Sigma_{1,2 n-1}-\Sigma_{2,2 n-1}-\ldots-\Sigma_{n, 2 n-1} .
$$

Proof. Suppose that the summands in $S$ with $N \leq k$ pairs of $D_{i}$ are counted correctly in $S^{(k)}=-\Sigma_{1,2 n-1}-\ldots-\Sigma_{k, 2 n-1}$. Then every summand with $N=k+1$ pairs of $D_{i}$ is "contained" in $\Sigma_{i}$ with the multiplicity $C_{k+1}^{i}$ and with the $\operatorname{sign}(-1)^{i-1} i \leq k$.

Hence we add to $S^{(k)}$

$$
(-1) \cdot\left(\sum_{i=1}^{k}(-1)^{i} C_{k+1}^{i}\right) \Sigma_{k+1,2 n-1}+(-1)^{k+1} \Sigma_{k+1,2 n-1}
$$

It is clear that in the sum $S^{(k)}+(22)$ all terms with $N \leq k+1$ pairs of $D_{i}$ are correctly calculated; on the other hand, $(22)=-1 \Sigma_{k+1,2 n-1}$ by the binomial formula for $(1-1)^{k+1}=0$.

Corollary. For $D_{1}, \ldots, D_{2 n} \in \mathfrak{A}$,

$$
\underset{A, D}{\operatorname{Alt}} \operatorname{Tr}\left(\left[D_{1}, A_{1}\right] \cdot \ldots \cdot\left[D_{2 n}, A_{2 n}\right] \cdot A_{2 n+1}\right)+\Sigma_{1,2 n-1}+\ldots+\Sigma_{n, 2 n-1}
$$

is a cocycle on the Lie algebra $\mathfrak{A}$.

Proof. Follows from Lemma 4.2.

Remark. It follows from Remark 4.2 that this cocycle is cohomologous to 0. 
4.6 .

Main Conjecture (Second Version). Let $\mathfrak{A}$ be an associative algebra with $\operatorname{Tr}, D_{1}, \ldots$, $D_{2 n}$ be its derivations satisfying conditions (i)-(iii) from Sect. 2.4. Then (in the notations of Sect. 4.4, 4.5)

$$
\begin{aligned}
\Psi_{2 n+1}\left(A_{1}\right. & \left., \ldots, A_{2 n+1}\right)= \\
& =\operatorname{Alt}_{A, D} \operatorname{Tr}\left(D_{1} A_{1} \cdot \ldots \cdot D_{2 n} A_{2 n} \cdot A_{2 n+1}\right)+\Sigma_{1,2 n-1}+\Sigma_{2,2 n-1}+\ldots+\Sigma_{n, 2 n-1}
\end{aligned}
$$

is a cocycle on the Lie algebra $\mathfrak{A}$ (and, hence, it solves the lifting problem).

Remark. Let $\mathfrak{A}$ be an associative algebra, $\mathfrak{h}$ be a two-sided ideal in $\mathfrak{A}, \quad \operatorname{Tr}-$ a trace on $\mathfrak{h}, \mathfrak{A}$ acts on $\mathfrak{h}$ by the adjoint action (see Ex. 2.3.2). Then the above formula is true without conditions (ii)-(iii) from Sect. 2.4, but any cocycle obtained in this way is cohomologous to 0 .

\section{7.}

Example $(n=3)$. In $\S 1$ we obtained the lifting formula for Dif ${ }_{1}$, in $\S 3$ we found it for Dif $_{2}$. Main Conjecture 4.6 states that the following formula is a lifting formula for $\mathrm{Dif}_{3}$ :

$$
\begin{aligned}
& \Psi_{3}\left(A_{1}, \ldots, A_{7}\right)=\operatorname{Alt}_{A, D} \operatorname{Tr}\left\{D_{1} A_{1} \cdot \ldots \cdot D_{6} A_{6} \cdot A_{7}\right. \\
& \left.+A_{1} \cdot Q_{12} \cdot A_{2} \cdot D_{3} A_{3} \cdot D_{4} A_{4} \cdot D_{5} A_{5} \cdot D_{6} A_{6} \cdot A_{7}\right] \\
& +D_{1} A_{1} \cdot A_{2} \cdot Q_{23} \cdot A_{3} \cdot D_{4} A_{4} \cdot D_{5} A_{5} \cdot D_{6} A_{6} \cdot A_{7} \\
& +D_{1} A_{1} \cdot D_{2} A_{2} \cdot A_{3} \cdot Q_{34} \cdot A_{4} \cdot D_{5} A_{5} \cdot D_{6} A_{6} \cdot A_{7} \quad \text { terms, linear in } Q_{i j} \\
& +D_{1} A_{1} \cdot D_{2} A_{2} \cdot D_{3} A_{3} \cdot A_{4} \cdot Q_{45} \cdot A_{5} \cdot D_{6} A_{6} \cdot A_{7} \\
& +D_{1} A_{1} \cdot D_{2} A_{2} \cdot D_{3} A_{3} \cdot D_{3} A_{4} \cdot A_{5} \cdot Q_{56} \cdot A_{6} \cdot A_{7} \\
& \left.+A_{1} \cdot Q_{12} \cdot A_{2} \cdot A_{3} \cdot Q_{34} \cdot A_{4} \cdot D_{5} A_{5} \cdot D_{6} A_{6} \cdot A_{7}\right] \\
& +A_{1} \cdot Q_{12} \cdot A_{2} \cdot D_{3} A_{3} \cdot A_{4} \cdot Q_{45} \cdot A_{5} \cdot D_{6} A_{6} \cdot A_{7} \\
& +A_{1} \cdot Q_{12} \cdot A_{2} \cdot D_{3} A_{3} \cdot D_{4} A_{4} \cdot A_{5} \cdot Q_{56} \cdot A_{6} \cdot A_{7} \\
& +D_{1} A_{1} \cdot A_{2} \cdot Q_{23} \cdot A_{3} \cdot A_{4} \cdot Q_{45} \cdot A_{5} \cdot D_{6} A_{6} \cdot A_{7} \\
& +D_{1} A_{1} \cdot A_{2} \cdot Q_{23} \cdot A_{3} \cdot D_{4} A_{4} \cdot A_{5} \cdot Q_{56} \cdot A_{6} \cdot A_{7} \\
& +D_{1} A_{1} \cdot D_{2} A_{2} \cdot A_{3} \cdot Q_{34} \cdot A_{4} \cdot A_{5} \cdot Q_{56} \cdot A_{6} \cdot A_{7} \\
& \left.\left.+A_{1} \cdot Q_{12} \cdot A_{2} \cdot A_{3} \cdot Q_{34} \cdot A_{4} \cdot A_{5} \cdot Q_{56} \cdot A_{6} \cdot A_{7}\right\}\right] \text { term, cubic in } Q_{i j}
\end{aligned}
$$

Recall that we don't alternate symbols $i, j$ in $Q_{i j}$.

\section{REFERENCES}

[A] Adler M. On a trace functional for formal pseudo-differential operators and the symplectic structure of the Korteweg - de Vries type equations, Invent. Math. 50 (1979) 219-248.

$[\mathrm{F}] \quad$ Feigin B.L. Lie algebra $\mathfrak{g l}(\lambda)$ and cohomology of a Lie algebra of differential operators, Russian Mathematical Surveys 43(2) (1988) 169-170.

[FT] Feigin B.L., Tsygan B.L. Cohomology of the Lie algebra of the generalized Jacobian matrices (in Russian), Funct. Anal. Appl. 17(2) (1983), 86-87.

[Fu] Fuks D.B. Cohomology of Infinite-Dimensional Lie Algebras, Consultants Bureau, New York and London, 1986.

[KK] Kravchenko O.S., Khesin B.A. Central extensions of the algebra of pseudo-differential symbols, Fuct. Anal. Appl. 25(2) (1991) 83-85. 
IUM, 11 Bol'shoj Vlas'evskij PeR., Moscow 121002, Russia

E-mail address: borya@main.mccme.rssi.ru 